\title{
Computer-Aided Image-Guided Bone Fracture Surgery: Modeling, Visualization, and Preoperative Planning
}

\author{
L. Tockus ${ }^{1,4}$, L. Joskowicz ${ }^{1}$, A. Simkin ${ }^{2}$, and C. Milgrom ${ }^{3}$ MD \\ 1 Institute of Computer Science, The Hebrew Univ., Jerusalem 91904 Israel. \\ 2 Lab. of Experimental Surgery, Hadassah Univ. Hospital, Jerusalem 91120 Israel. \\ 3 Dept. of Orthopaedic Surgery, Hadassah Univ. Hospital, Jerusalem 91120 Israel. \\ 4 Biomedicom, Technology Park, Malha, Mail Stop IIL 376 Jerusalem 91487, Israel \\ lana@biomedicom.co.il, josko@cs.huji.ac.il \\ ruskin@vms.huji.ac.il,milgrom@md2.huji.ac.il
}

\begin{abstract}
This paper describes the Fracas computer-integrated orthopaedic system for assisting surgeons in closed long bone fracture reduction. Fracas' goals are to reduce the surgeon's cumulative exposure to radiation and improve the positioning accuracy by replacing uncorrelated static fluoroscopic images with a virtual reality display of $3 \mathrm{D}$ bone models created from preoperative CT and tracked intraoperatively in real-time. Fluoroscopic images are used to register the bone models to the intraoperative situation and to verify that the registration is maintained. This paper describes the system concept, and the software prototypes of the modeling, preoperative planning, and visualization modules.
\end{abstract}

\section{Introduction}

Fluoroscopy-based orthopaedic procedures crucially depend on the ability of the surgeon to mentally recreate the spatio-temporal intraoperative situation from uncorrelated, two-dimensional fluoroscopic X-ray images. Significant skill, time, and frequert use of the fluoroscope are required, leading to positioning errors and complications in a non-negligible number of cases, and to significant cumulative radiation exposure of the surgeon [12]. Recent research shows that computeraided systems can significantly improve the accuracy of orthopaedic procedures by replacing fluoroscopic guidance with interactive display of 3D bone models created from preoperative CT studies and tracked in real time. Examples include systems for acetabular cup placement [13], for total knee replacement, and for pedicle screw insertion $[5,8]$.

We are currently developing a computer-integrated orthopaedic system, called FRACAS, for closed medullary nailing of long bone fractures. Medullary nailing is a very frequent routine procedure that restores the integrity of the fractured bone by means of a nail inserted in the medullary canal [1]. The nail is placed without surgically exposing the fracture through an opening close to the piriformus fossa in the proximal femoral bone. The surgeon manually aligns the bone 

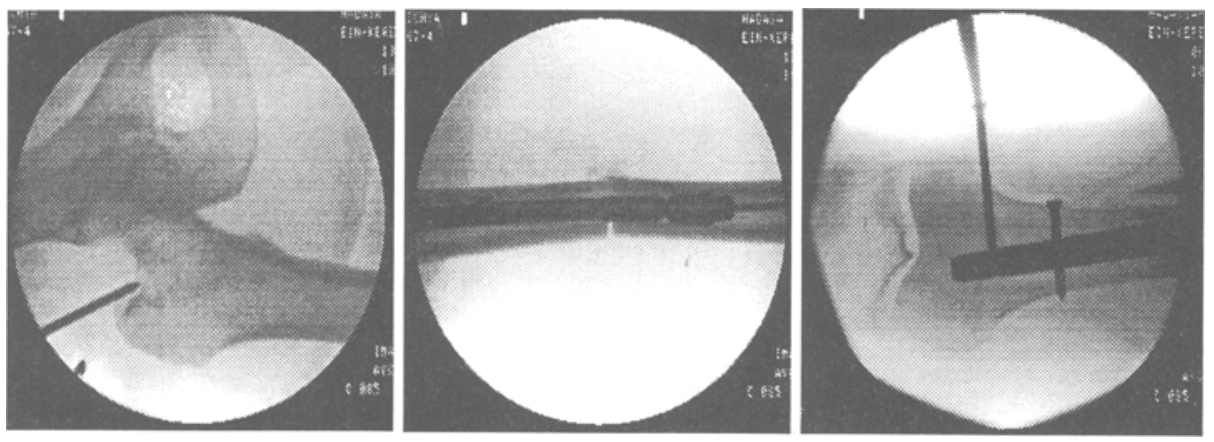

Fig. 1. Fluoroscopic images showing the steps of closed medulary nailing (from left to right): opening of the medulary canal; alignement of the bone fragments and nail insertion; and distal locking.

fragments, inserts a guide wire, reams the canal if necessary, and drives the nail in with a hammer. Lateral proximal and distal interlocking screws are inserted to prevent fragment rotation and bone shortening. All these steps are performed under fluoroscopic guidance (Fig. 1).

The most common errors and complications in closed medullary fixation come from limited viewing capabilities. Because all alignment, reaming, and positioning must be done under fluoroscopy, the surgeon must mentally reconstruct the location of the parts in space and time, manipulate the tools and the bone without visual feedback, and confirm the position with a new set of fluoroscopic images. This often imprecise, slow, and tedious procedure can cause improper positioning and alignment, inadequate fixation, malrotation, bone cracking, cortical wall penetration, bone weakening with multiple or enlarged screw holes in the worst case. The surgeon's direct exposure to radiation in each procedure is between 3 to 30 min with $31-51 \%$ spent on distal locking [12] depending on the patient and the surgeon's skill. When the bone fragments are difficult to align, the surgeon reverts to open techniques. The surgeon's radiation exposure and the reported number of complications of this kind motivates the search for improved solutions.

FracAs' goals are to reduce the surgeon's cumulative exposure to radiation and improve the positioning and navigation accuracy by replacing uncorrelated static fluoroscopic images with a virtual reality display of $3 \mathrm{D}$ bone models created from preoperative CT and tracked intraoperatively in real-time (Figure 2). Fluoroscopic images are used for registration - to establish a common reference frame - between the bone models and the intraoperative situation, and to verify that the registration is maintained. This paper describes the system concept and the software prototypes of the modeling, preoperative planning, and visualization, modules. A companion paper describes fluoroscopic image processing. 


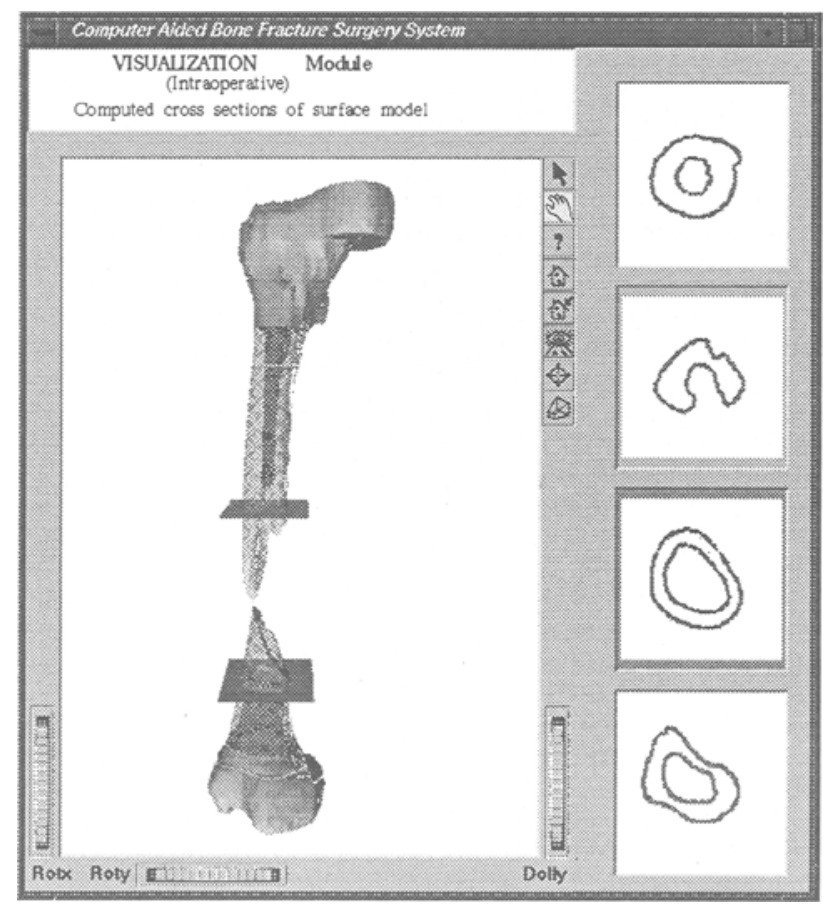

Fig. 2. Intraoperative three-dimensional distal and proximal bone fragment models that replace the fluoroscopic images, as displayed by the Visualization module. Their location is tracked and updated in real time during surgery. The four windows on the right show the inner and outer bone contours at the selected cross sections corresponding to planes on the left.

\section{Previous work}

Two computer-based systems for long bone fracture reduction $[10,15]$ and $[3]$ focus on distal screw locking. Both use intraoperative fluoroscopy and optical tracking without preoperative CT images. $[10,15]$ uses a passive mechanical arm with optical encoders to guide the surgeon to the right drilling position. It automatically identifies the distal holes in the fluoroscopic images, plans the drilling trajectory, and constraints passive arm motions. The advantage of the system is that it eliminates trial-and-error drill positioning. The disadvantage is that it requires additional mechanical hardware. [3] continuously displays the projection of the surgical tools as they move on preselected fluoroscopic images, thus approximating continuous fluoroscopy. Since the images are correlated, the surgeon can simultaneously view the tool's progression from several viewpoints. This is similar to the conventional procedure and reduces the number of images needed. However, it does not provide preoperative planning support, does not display $3 \mathrm{D}$ views, and does not update the bone fragment positions in real time. 


\section{Goals and rationale}

Our goal in developing FracAs is to assist the surgeon in all the steps of fracture reduction, not just in distal locking. It provides $3 \mathrm{D}$ bone modeling, preoperative planning, fluoroscopic image processing, and anatomy-based registration of the $3 \mathrm{D}$ model with the fluoroscopic images.

We believe that the additional cost and time of the preoperative CT study is outweighed by the potential reduction in more expensive intraoperative time, in minimizing mistakes and repetitive attempts at fracture reduction, and in reducing the surgeon's exposure to radiation. In many centers, including ours, CT is readily available for trauma patients, adding little preoperative time, risk, and morbidity. Cases of fracture femur may be divided into those in which the sole injury is the fractured femur, and those which there is multiple trauma. Multiple trauma patients are likely to have other CT studies done anyhow, e.g., abdomen and pelvis. In both cases, the patient's leg is immobilized on a Thomas splint which makes transport on the emergency room stretcher and transfer to the CT table easy and relatively pain free, with minimal fracture movement. Any fracture movement that does occur would not be associated with an increased risk for pulmonary emobilism.

\section{FRACAS: system description}

The Fracas system [4] comprises three units: a standard fluoroscopic C-arm, a real-time position tracking optical system, and a computer workstation with data processing and visualization software. The fluoroscopic unit is used to capture images that are used to establish a common reference frame (registration) between the intraoperative bone position and preoperative bone fragment models. The tracking unit is used to provide accurate, real-time spatial object positions with optical cameras following infrared light-emitting diodes (LEDs) rigidly mounted on the surgical instruments or attached to the bones via bone screws [5, 9]. The computer workstation is used preoperatively for modeling and planing, and intraoperatively for data fusion and display.

The envisaged sequence of the procedure is as follows: preoperatively, a CT of the healthy and fractured bones is acquired. Surface and canal bone models are constructed by the modeling module. Using the planning modules, the surgeon interactively selects the distal and proximal bone fragments and nail type, length, and diameter. Shortly before the surgery, the fluoroscopic and tracking units are calibrated by a technician. The patient is then brought into the operating room and the surgeon prepares and exposes the femur canal following the usual procedure.

To track the position of the bone fragments, the surgeon rigidly implants two specially prepared bone screws with LEDs attached to them. Fluoroscopic images are then captured by a technician (without the nearby presence of the surgeon) to perform an anatomy-based registration with the preoperative bone fragment models. With the LEDs in place, the technician activates the optical tracking 


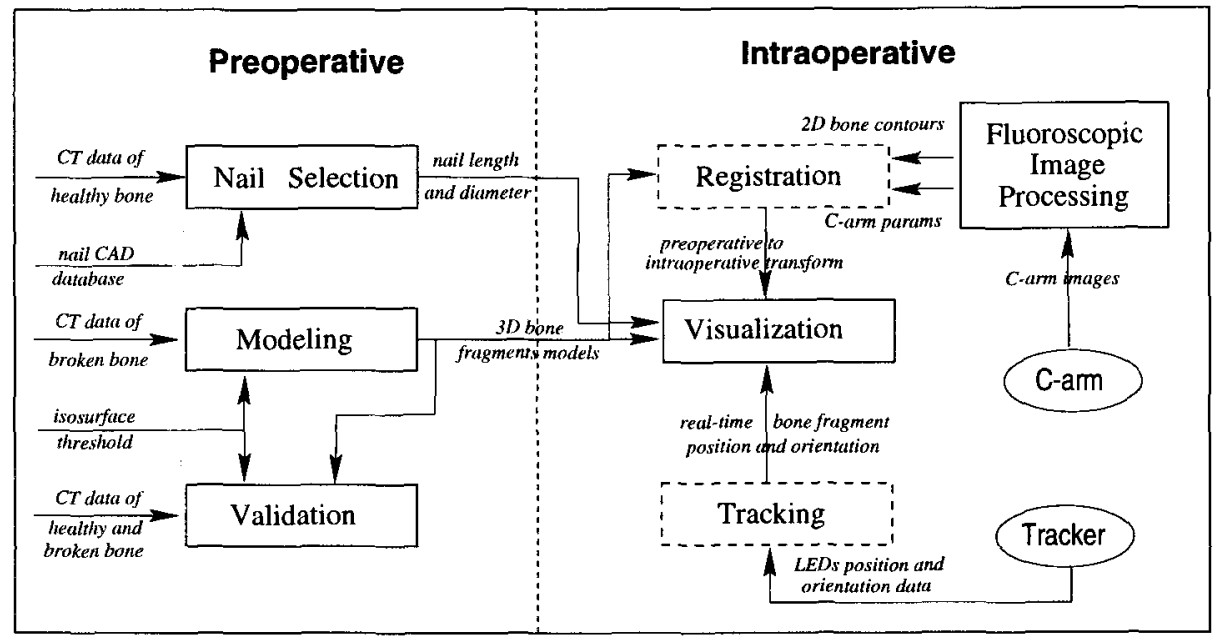

Fig. 3. Flow diagram of FraCAS's main software modules. Solid rectangles are implemented modules, dashed ones are under construction.

system to capture the location of the bone fragments and surgical instruments. The images are imported into the workstation via a video frame grabber, and the tracking data via a serial port. The visualization module constructs the virtual reality image showing the bone fragments and surgical instrument models and presents it to the surgeon on a high-definition monitor. The surgeon manipulates the bone fragments and surgical tools, following their relative positions and orientations on the monitor. Once the desired positioning is achieved, a new set of fluoroscopic images is captured and registered to confirm the intraoperative situation with the displayed model. This process is repeated for the different steps of the procedure.

Figure 3 shows the main FracAs software modules and the data flow between them. We describe the modeling and validation, nail selection, and visualization modules in detail next. The fluoroscopic image processing module is presented in [16]. The registration and tracking modules are under development.

\section{$5 \quad$ Modeling}

The modeling module inputs the CT data and a user-defined bone density threshold value. It outputs the inner and outer surface models of the selected proximal and distal bone fragments. The models are produced in two steps. First, polygonal surface models of the bone external and internal fragment surfaces are created. Then, the surgeon interactively defines the extent of the proximal and distal fragments of interest with cutting planes. Figure 4 shows the modeling module window with a segmental comminuted fracture.

The bone fragment surface models are meshes of connected triangles. They are extracted from the CT data using an extended Marching Cubes algorithm 


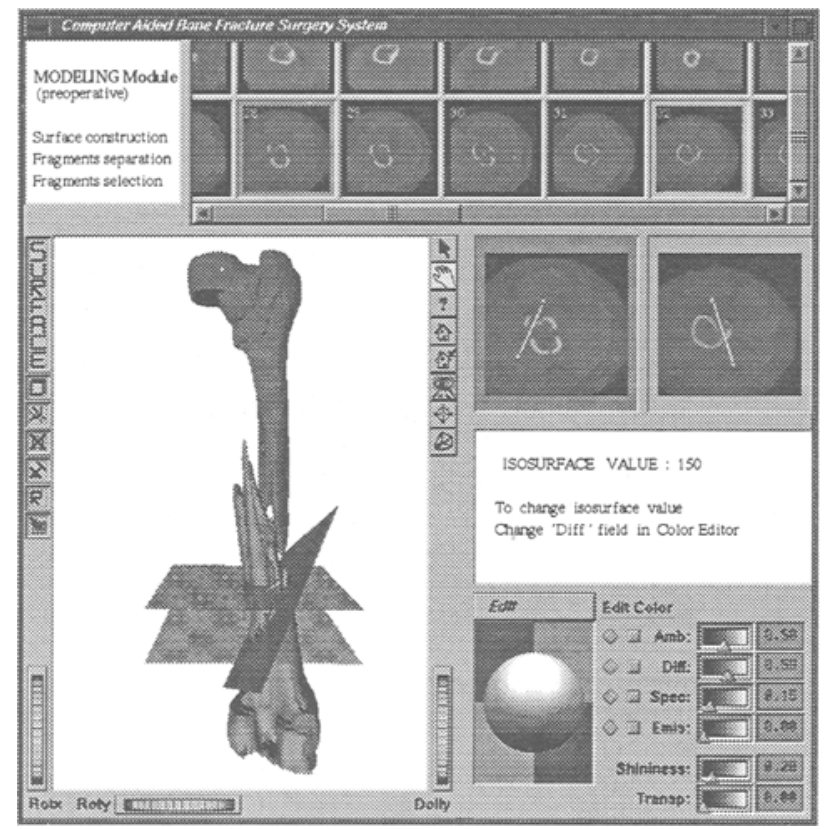

Fig. 4. The Modeling module display. The top window shows the original CT slices. The left bottom window shows the surface model of the fractured bone (the canal surface is hidden). The horizontal planes indicate the position of the selected CT slices shown in detail on the two windows on the right. The slanted plane is the user-defined cutting plane that separates the bone fragments. The bottom right window shows the bone density threshold and the material and viewing parameters.

[7] which classifies surface triangles as proximal, distal, canal, or outer surface. The size of the triangular meshes depends on the CT data, typically consisting of several tens of thousands of triangles. For example, the CT set in Figure 4 consists of 44 slices at $10 \mathrm{~mm}$ intervals, image size $160 \times 160$, pixel size $1 \mathrm{~mm}^{2}$; it yields a model of about 75,000 triangles.

Since the bone fragments are usually in contact due to the action of the muscles, bone and canal surface models are frequently lumped together (a single connected piece for linear fractures, several pieces for segmental and comminuted fractures). An additional step is necessary to separate the fragment models because threshold information alone is not sufficient to determine when a new fragment begins and when one ends when they are in contact.

The fragment models are separated into different pieces by means of userdefined cutting planes whose intersection defines regions of interest. Cutting planes can be positioned in one of two ways. One is by defining the plane in a default configuration and bringing it to the desired location using manipulation dials (on the left and bottom part of the window in Figure 4). The drawback is 
that the cutting plane is initially in an arbitrary position and might require a series of non-intuitive dial manipulations to bring it to the desired position.

A more suitable method is to interpolate a cutting rectangle from two line segments defined in two CT slices close to the area of the desired separation (the white line segments in the two detailed CT windows on the right in Figure 4). To avoid separating model parts that are far from the cutting area, the plane is automatically clipped to a rectangle. The position of the resulting cutting plane can be interactively fine-tuned by changing the position of the segment endpoints or by using the manipulation dials. This procedure is repeated until one or more planes are in the desired position. The fragments are then separated according to their point membership classification relative to each plane. Undesired bone fragments and bone particles are eliminated by clicking on them with the mouse. To differentiate between them, the selected bone fragments are displayed in different colors (dark upper fragment in Figure 4).

To verify that the computed surface model closely matches the CT data, we developed a visual validation module. For each CT slice, the module computes the cross section of the geometric model at the CT slice location and shows it superimposed with the original CT data. The discrepancy between the two can be determined by visual inspection by moving up and down the CT slices. Quantitative measures could be computed if necessary. To improve the fit, the bone density threshold value can be adjusted accordingly.

\section{Nail selection}

The nail selection module assists the surgeon to select the optimal nail size and length when a CT of the healthy bone is available. It inputs the CT data of the healthy bone and a predefined CAD library of nail models available on site. Its output is the universal nail type, its length, and its diameter. The module constructs a bone and canal surface model and allows the surgeon to perform spatial measurements on it. Figure 5 shows the nail selection window.

To select a nail, the surgeon performs a series of canal diameter and height measurements. Canal diameter measurements are performed on the CT slices (four right windows in Figure 5) by moving the endpoints of a measuring segment (shown in white). Its length is interactively updated and appears in the top left corner of the window. Height measurements are performed on the 3D window (left window) by moving the endpoints of the measuring segment (black segment). Its length is interactively updated and appears in the top left corner of the window. Having determined the closest standard diameter and nail length, the surgeon enters the nail size, and the system retrieves the corresponding CAD model from the nail library. Nail diameters typically come in intervals of $1 \mathrm{~mm}$, lengths in intervals of $20 \mathrm{~mm}$. The nail model is then interactively positioned with the manipulator dials inside the bone to ensure that the diameter is adequate and that there is no impingement with the knee or hip joint. Although the actual nail deforms when it is inserted into the canal, the displayed nail position is close enough for the preoperative evaluation and selection. 


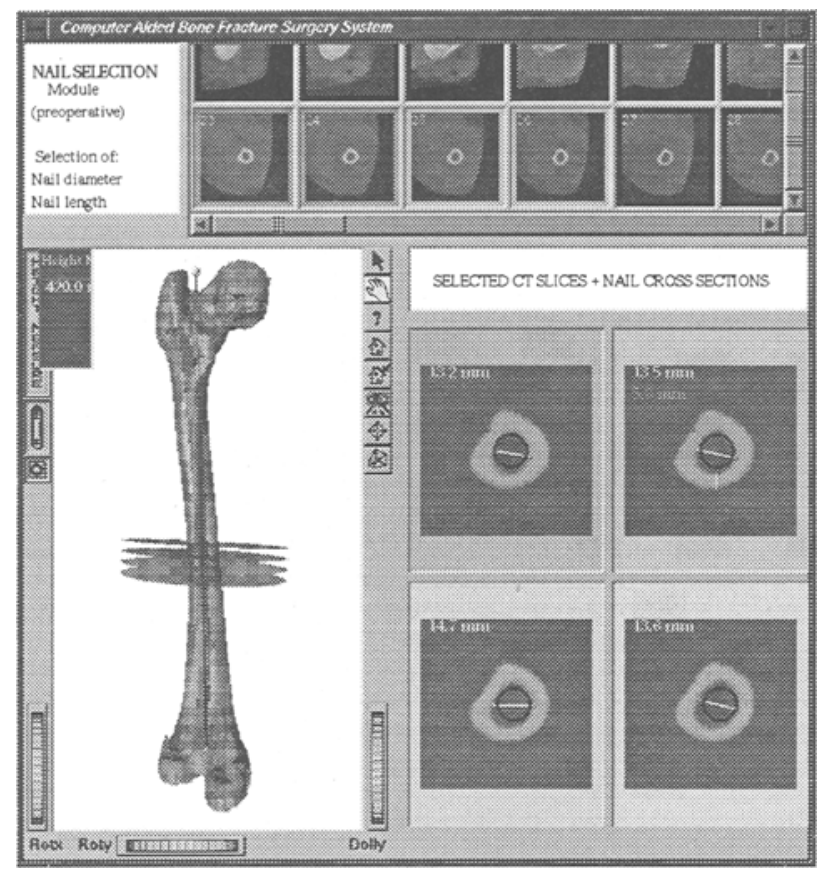

Fig. 5. The Nail Selection module display. The top window shows the original CT slices. The left bottom window shows the healthy bone surface model, the selected nail model, and the planes of the four selected CT slices shown in detail on the four windows to the right of it. The detailed CT slices windows on the right show the nail cross section (dark circle) superimposed on the original CT image.

\section{$7 \quad$ Visualization}

The visualization module provides the surgeon with a virtual reality view of the intraoperative position and orientation of the proximal and distal fragments, the nail, and the surgical instruments. The positions are updated with real-time data from the tracking unit, after the preoperative model and the intraoperative situation have been registered (Figure 2). The inputs are the proximal and distal fragment models, the nail model, the registration transformation, and the on-line tracking position information. The output is a display of the updated virtual reality model, which can be seen from any desired perspective, as illustrated in Figure 2 (the nail is omitted; models of locking screws and other surgical instruments can be readily incorporated).

Since the main task is to align the proximal and distal canal, FraCAS displays the central part of the exterior bone fragments translucid and highlights the canal surface inside. The femoral head and the condyles are displayed opaque to provide a reference frame for the joint and bone axes. Note that because of the fracture, the canal can vanish altogether before the end of the fragment. The virtual reality window provides standard viewing capabilities, e.g., zooming, 
viewpoint and perspective modification. It also includes four windows for cross section visualization, which can be interactively moved up and down the bone (note that the original CT image cannot be displayed, as the bone fragment position has changed).

\section{Conclusion}

We have described the design and implementation of the modeling, nail selection, and visualization modules of the FrACAS computer-integrated system for closed medullary nailing. The goal of system is to overcome most of the planning, viewing and spatial positioning difficulties associated with orthopaedic imageguided surgery. Fracas constructs a 3D model of the exterior and canal surface bone fragments and displays an updated view of their position and orientation. It assists surgeons in selecting the optimal nail length and diameter by interactively positioning a CAD nail model inside the healthy bone model. Its main advantages are spatial display and real-time image update, akin to continuous fluoroscopy. To date, we have conducted evaluation trials on five patient data sets to test the user interface and validate the prototype.

Current work on the Fracas system includes fluoroscopic image processing (image dewarping, C-arm calibration, and contour extraction) [16] and anatomic $2 \mathrm{D} / 3 \mathrm{D}$ registration. Near term plans include real-time tracking, system integration and in-vitro prototype testing.

\section{Acknowledgments}

Leo Joskowicz is supported by a Guastalla Faculty Fellowship. Lana Tockus was supported by a Silicon Graphics Biomedical (now Biomedicom) grant.

\section{References}

1. Brumback R.J., "Regular and Special Features - The Rationales of Interlocking Nailing of the Femur, Tibia, and Humerus" Clinical Orthopaedics and Related Research, 324, Lippincott-Raven, 1996.

2. Fadda M. Bertelli, D., Martelli S. Marcacci, M., Dario P., Carmella D. and Trippi D., "Computer Assisted Planning for Total Knee Arthroplasty" CVRMed-MRCAS'97, Lecture Notes in Computer Science 1205, Troccaz et al. eds., Springer 1997.

3. Hofstetter, R., Slomczykowski, M. Bourquin, I, Nolte, L.P, "Fluoroscopy Based Surgical Nagivation - Concept and Clinical Applications", Proc. 11th Int. Symp. on Computer Assisted Radiology and Surgery, H.U. Lemke, et al. eds, Springer 1997.

4. Joskowicz, L., Tockus, L., Yaniv, Z, Simkin, A., Milgrom, C. "Computer-Aided Image-Guided Bone Fracture Surgery - Concept and Implementation", Proc. 12th Int. Symp. on Computer Assisted Radiology and Surgery, H.U. Lemke et. al. eds, 1998.

5. Lavallée, S., Sautot, P., Troccaz, J., Cinquin, P., Merloz, P. "Computer Assisted Spine Surgery: a Technique for Accurate Transpedicular Screw Fixation Using CT Data and a 3D Optical Localizer", J. of Image-Guided Surgery 1(1), 1995. 
6. Leitner F., Picard F., Minfelde R., Schultz H.J., Cinquin P. and Saragaglia D., "Computer-assisted knee surgical total replacement" CVRMed-MRCAS'97, Lecture Notes in Computer Science 1205, Troccaz et al. eds., Springer 1997

7. Lorensen W.E. and Cline H.E., "Marching Cubes: A High Resolution 3D Surface Construction Algorithm", SIGGRAPH'87, Vol. 21(4), July 1987.

8. Li Q.H., Holdener H.J., Zamorano L., et. al. Nolte L.P., Visarius H., and Diaz F., "Computer-assisted transpedicular screw insertion" Lecture Notes in Computer Science, Springer, 1996.

9. Nolte, L.P., Zamorano L., et. al. "A Novel Approach to Computer Assisted Spine Surgery", Proc. 1st Int. Symp. on Medical Robotics and Computer Assisted Surgery, Pittsburgh, 1994.

10. Phillips R., Viant W.J., Mohsen A.M.M.A., Griffits J.G., Bell M.A., Cain T.J., Sherman K.P. and Karpinski M.R.K., "Image Guided Orthopaedic Surgery - Design and Analysis" IEEE Transactions on Robotics and Control, March 1996.

11. Russel T.A., "Fractures of Lower Extremity" Campbell's Orthopaedic Encyclopedia, 1995.

12. Sanders R., "Exposure of the Orthopaedic Surgeon to Radiation", J. of Bone Joint Surgery 75 A(3), 1993.

13. Simon, D.A, Jaramaz, B, Blackwell, et al. "Development and Validation of a Navigational Guidance System for Acetabular Implant Placement", Proc. of CVRMedMRCAS'97, Lecture Notes in Computer Science 1205, Springer 1997.

14. Tockus, L. "A System for Computer-Aided Image-Guided Bone Fracture Surgery: Modeling, Visualization, and Preoperative Planning", Msc Thesis, Hebrew U., Jerusalem, July 1997.

15. Viant, W.J., Phillips, R., Griffiths, et al. "A Computer Assisted Orthopaedic System for Distal Locking of Intramedullary Nails", Proc. of MediMEC'95, 1995.

16. Yaniv, Z., Joskowicz, L., Simkin, A. Garza-Jinich, M, Milgrom C. "Fluoroscopic Image Processing for Computer-Aided Orthopaedic Surgery" 1st Int. Conf. on Medical Computing and Computer-Assisted Intervention, Boston, 1998. 